\title{
Characteristics of breast metastases from non-breast solid tumors in 22 patients from a southern Chinese population
}

\author{
PENG SUN, JIEWEI CHEN, JIABIN LU, RONGZHEN LUO, MEI LI and JIEHUA HE \\ State Key Laboratory of Oncology in South China, Department of Pathology, Sun Yat-sen University Cancer Center, \\ Collaborative Innovation Center for Cancer Medicine, Guangzhou, Guangdong 510080, P.R. China
}

Received September 11, 2016; Accepted November 7, 2017

DOI: $10.3892 / \mathrm{ol} .2018 .7741$

\begin{abstract}
Breast metastases from solid non-breast tumor types are rare; however, they should be always considered in the differential diagnosis of a breast lesion owing to the associated poor patient prognosis and the requirement of different therapeutic strategies compared with those used to treat primary breast cancer. The aim of the current study was to summarize the characteristics of metastases to the breast in a southern Chinese population. The medical records and pathological sections of 22 patients with pathologically confirmed extra-mammary metastases to the breast that presented to Sun Yat-sen University Cancer Center between January 2000 and December 2015 were retrospectively reviewed. The median age of onset for breast metastasis was 43 years (range, 10-62 years) and $19(86.4 \%)$ patients had a known history of a primary tumor. The mean interval from diagnosis of the primary tumor to breast metastasis was 16.5 months (range, 6-56 months). A unilateral (45.5\% left, $36.4 \%$ right), upper outer quadrant $(15 / 22,68.2 \%)$ lesion of the breast was most frequently initially detected by self-checking $(63.6 \%)$. The most common origin of the primary tumor was the lung (22.7\%). Nasopharyngeal carcinoma accounted for a high proportion of the metastases (18.2\%). The median duration of survival from the time of diagnosis of a breast metastasis was 14 months (range, 2-74 months). A total of 10 patients $(45.5 \%)$ succumbed to the disease. The results also indicated that overall survival in patients that underwent surgery was improved compared with patients who did not undergo surgery. The results of the present study demonstrated that clinical history, imaging findings, pathology from the primary tumor and immunostaining were required
\end{abstract}

Correspondence to: Dr Jiehua He, State Key Laboratory of Oncology in South China, Department of Pathology, Sun Yat-sen University Cancer Center, Collaborative Innovation Center for Cancer Medicine, 651 Dongfeng East Road, Guangzhou, Guangdong 510080, P.R. China

E-mail: hejh@sysucc.org.cn

Key words: breast metastases, clinical characteristics, pathological classification, prognosis, immunohistochemical staining in combination to establish an accurate diagnosis. Further investigation into the improvement of the prognosis of patients with metastases to the breast following surgery is required.

\section{Introduction}

Breast cancer is one of the most common malignant tumors in females, while metastases to the breast from non-breast solid tumors are rare, accounting for only $0.3-2 \%$ of all malignant mammary tumor types $(1,2)$. To date, $<500$ cases of cancer with secondary involvement of the breast from any non-breast solid tumors have been reported $(2,3)$. On the basis of the limited number of studies available in the literature, excluding contralateral breast cancer and lymphoma, the most frequent source of primary malignancies with metastases to the breast in Western countries are melanoma and lung cancer (3-5). Other reported primary sources include the ovary, gastrointestinal tract, thyroid, kidney and sarcomas of different origins (6-8). In general, symptoms of metastases to the breast are similar to those of primary breast cancer, including the presence of a palpable, freely-movable mass within the breast. Pain, tenderness and inflammation are also observed occasionally. Considering the rarity of extramammary lesions, it is challenging to distinguish these lesions from primary breast cancer, even in patients with a history of a primary non-breast solid tumor (1-4). However, the poor prognosis of patients with secondary breast metastases and the contrast in appropriate treatments compared with those for patients with primary breast cancer, in addition to the fact that systemic treatment or palliative care is more appropriate than extensive surgery in the majority of patients with secondary breast metastases, emphasizes the importance of accurate diagnosis (1-6). In the present study, a single-institution retrospective review of 22 patients with pathologically confirmed extramammary metastases to the breast, treated at the Sun Yat-sen University Cancer Center (Guangzhou, China), was conducted. The clinical, radiological, pathological and prognostic data of these patients were summarized in order to identify their clinical characteristics, describe their histological and immunohistochemical features and to assess their clinical outcomes. The results may serve as a future reference for the Southern Chinese population. 


\section{Patients and methods}

Patients. Retrospective data were obtained from electronic medical records and pathology databases at the Sun Yat-sen University Cancer Center (Guangzhou, China) between January 2000 and December 2015 for patients with biopsy-diagnosed metastasis to the breast from an on-breast primary solid tumor site. Tissues were also obtained within the same time frame. Metastases from contralateral primary breast cancer or hematological malignancies were excluded. All histological slices were reviewed by two pathologists.

Medical records from 22 patients ( 2 males, 20 females; mean age, 40.5 years; range, $10-62$ years) were included in the present study. Data collected from the records included the following: Age, sex, initial symptoms, history of primary tumor, interval from the diagnosis of the primary tumor to breast metastases, other metastases status, tumor status at presentation of breast metastasis, ultrasonographic and mammographic findings, biopsy or post-surgery pathology results, clinical treatments and follow-up. The breast imaging-reporting and data system (BI-RADS) I to VI categorization (9), developed by the American College of Radiology, was applied in present study.

Statistical analysis. Descriptive statistics were applied to assess frequency distributions. Overall survival interval probabilities were calculated using the Kaplan-Meier method and differences in survival were assessed by the log-rank test using SPSS 19.0 (IBM Corp., Armonk, NY, USA). P<0.05 was determined to indicate statistically significant difference.

\section{Results}

Demographic characteristics of patients with metastases to the breast. Demographic characteristics of 22 patients with metastases to the breast are summarized in Table I. Patients were predominately female ( 2 males, 20 females). The mean and median onset of age for breast metastasis was 40.5 and 43 years, respectively (range, $10-62$ years). A total of 19 (86.4\%) patients had a documented history of a primary tumor with a mean interval of 6.5 months (range, 6-56 months) between diagnosis of the primary tumor to detection of breast metastasis. In the other 3 patients (13.6\%), the primary tumor and breast metastasis were diagnosed simultaneously with intact primary disease. A total of 7 patients $(31.8 \%)$ presented with other metastases and 11 patients (50.0\%) exhibited no evidence of a primary tumor or other metastasis when breast metastasis was located. Pulmonary metastasis had been confirmed and treated in one patient with melanoma when breast metastasis presented. Overall, the breast was the only metastatic site in $6(27.3 \%)$ patients.

Clinical characteristics of patients with metastases to the breast. Breast metastasis were initially detected by self-checking in 14 patients (63.6\%; Table II). The mean tumor size was $2.9 \mathrm{~cm}$ (range, $0.8-12.0 \mathrm{~cm}$ ). A unilateral (45.5\% left, $36.4 \%$ right), upper outer quadrant (15/22, $68.2 \%$ ) lesion of the breast was most frequently diagnosed; bilateral lesions were present in 4 patients $(18.2 \%)$. Clinically, 14 patients $(63.6 \%)$ presented with a palpable
Table I. Demographic characteristics of patients with metastases to the breast $(\mathrm{n}=22)$.

\begin{tabular}{lc} 
Characteristics & Patients, $\mathrm{n}(\%)$ \\
\hline Sex & $2(9.1)$ \\
Male & $20(90.9)$ \\
Female & \\
Age, years & 40.5 \\
Mean & 43 \\
Median & $10-62$ \\
Range & \\
History of primary tumor & $19(86.4)$ \\
Known & $3(13.6)^{\mathrm{a}}$ \\
Unknown & \\
Interval from primary tumor to & \\
breast metastasis & 16.5 \\
Mean, months & $6-56$ \\
Range, months & \\
Other metastases & $6(27.3)$ \\
Breast only & $9(40.9)$ \\
Other metastases prior to breast & $2(9.1)$ \\
Simultaneous other metastases & $5(22.7)$ \\
Other metastases following breast & \\
Tumor status at presentation of & \\
breast metastasis & \\
Metastatic disease & $1(31.8)$ \\
No evidence of disease & $1(4.5)$ \\
History of other metastases, no & \\
NED at present & $3(13.6)^{\mathrm{a}}$ \\
Intact primary disease &
\end{tabular}

ane patient was initially diagnosed with a double primary tumor (lung cancer and breast cancer), the breast lesion was pathologically diagnosed postoperatively (modified radical mastectomy) as a metastasis from the lung.

painless solitary mass, 8 (36.4\%) had multiple nodules, $1(4.5 \%)$ exhibited skin and nipple changes and $2(9.1 \%)$ reported tenderness. A total of $10(45.5 \%)$ patients had palpable enlarged axillary lymph nodes, of which 4 patients (18.2\%) presented with enlarged supraclavicular lymph nodes simultaneously.

Ultrasonic and mammographic findings of breast metastases. Among the 22 patients who underwent radiologic imaging (Table III), 14 patients underwent breast ultrasonography and 5 patients underwent a mammography. In mammography, the most common finding was a single mass with either circumscribed $(3 / 5,60 \%)$ or speculated $(2 / 5,40 \%)$ margins. Only $1(20 \%)$ patient who underwent a mammography presented with a lesion classified as a BI-RADS category I which refers to a negative examination by mammography, and the rest $(4 / 5,80 \%)$ presented lesions categorized as BI-RADS category IVb or greater, which refers to a high probability of malignancy. In ultrasonography, breast lesions were primarily 
Table II. Clinical characteristics of patients with metastases to the breast.

\begin{tabular}{lc}
\hline Characteristics & Patients, $\mathrm{n}(\%)$ \\
\hline Initial detections & \\
Self-checking & $14(63.6)$ \\
Ultrasonography & $2(9.1)$ \\
Mammogram & $2(9.1)$ \\
CT (including PET-CT) & $3(13.6)$ \\
Unknown & $1(4.5)$ \\
Clinical symptoms & \\
A palpable painless solitary mass & $14(63.6)$ \\
Multiple nodules & $8(36.4)$ \\
Skin and nipple changes & $1(4.5)$ \\
Tenderness & $2(9.1)$ \\
Enlarged axillary lymph nodes & b \\
Tumor size, cm & $10(45.5)$ \\
Mean & \\
Range & 2.9 \\
Breast involvement & $0.8-12$ \\
Unilateral, left & \\
Unilateral, right & $10(45.5)$ \\
Bilateral & $8(36.4)$ \\
Quadrant & $4(18.2)$ \\
Innerc: Upper/mid/lower & \\
Outer ${ }^{\mathrm{b}}$ U Upper/mid/lower & $18(69.2)$ \\
\hline
\end{tabular}

${ }^{\mathrm{a} N o}$ evidence of inflammatory skin changes. ${ }^{\mathrm{b}}$ Simultaneous enlarged supraclavicular lymph nodes in 4 cases. ${ }^{\mathrm{c}}$ Upper/middle/lower, 4/3/1, respectively. ${ }^{\mathrm{d}}$ Upper/middle/lower, $15 / 2 / 1$, respectively. CT, computerized tomography; PET-CT, positron emission tomography-computerized tomography.

hypoechoic and margins may be either circumscribed $(8 / 14$, $57.1 \%)$ or speculated $(6 / 14,42.9 \%)$. The vascularity $(8 / 14$, $57.1 \%)$ of the lesions and BI-RADS category V $(7 / 14,50 \%)$ were most commonly identified. Calcifications were observed in only 2 cases in ultrasonography (14.3\%) and mammography $(40 \%)$ each.

Histological features in different types of carcinoma and non-carcinoma in patients with breast metastases. Histological features of different tumor types and primary tumor sites are presented in Tables IV and V. Carcinoma was the most common tumor type for non-mammary metastases (16/22, 72.7\%; Table IV), followed by melanoma $(3 / 22,13.6 \%$; Table V) and sarcoma $(2 / 22,9.1 \%$; Table V). The remaining patient presented with a rare Wilms' tumor metastasis to the breast (Table V). The most frequent primary tumor site for carcinoma was the lungs $(5 / 22,22.7 \%$, Fig. 1), followed by the nasopharynx (4/22, 18.2\%; Fig. 2$)$ and ovary (3/22, 13.6\%; Fig. 3). Other primary sites included the gastrointestinal tract $(2 / 22,9.1 \%)$ and thyroid $(1 / 22,4.5 \%)$. Of the three melanoma cases, two initially occurred at cutaneous sites and one was ocular (Fig. 4 and Table V). The two cases of sarcoma were
Table III. Ultrasonic and mammographic findings of breast metastases $^{\mathrm{a}}$.

\begin{tabular}{lc} 
Characteristics & Patients, $\mathrm{n}$ \\
\hline Ultrasonic findings (n=14) & \\
Circumscribed/speculated margins & $8 / 6$ \\
Posterior echo & \\
No change/attenuation/enhancement & $8 / 3 / 3$ \\
BI-RADS category, II/III/IVa/IVb/IVc/V & $1 / 1 / 1 / 2 / 2 / 7$ \\
Calcifications & 2 \\
Cooper's ligaments involved & 1 \\
Skin/subcutaneous nodule & 3 \\
Vascularity & $8^{\mathrm{b}}$ \\
Mammographic findings (n=5) & \\
Margins, circumscribed/speculated & $3 / 2$ \\
BI-RADS category, I/IVb/V & $1 / 3 / 1$ \\
Calcifications & 2 \\
\hline
\end{tabular}

${ }^{a}$ Ultrasonic or mammographic imaging records were available in 18 cases; one only had a PET-CT scan, 3 patients unknown; ${ }^{\mathrm{b}}$ Abnormal blood flow signals detected by Doppler imaging either inside ( 7 cases) or surrounding ( 1 case) the lesion. BI-RADS, breast imaging-reporting and data system; PET-CT, positron emission tomography-computerized tomography.

rhabdomyosarcoma (Fig. 5), with the primary sites being the palm and nasal cavity (Table V).

Immunohistochemistry (IHC) was performed in 15 patients $(68.2 \%)$ in total. Of these, lung cancer was the most frequent tumor identified $(5 / 15,33.3 \%)$, followed by tumors of asopharynx $(3 / 15,20.0 \%)$, ovary $(2 / 15,13.3 \%)$, melanoma $(2 / 15,13.3 \%)$, rhabdomyosarcoma $(1 / 15,6.7 \%$; data not shown), Wilms' tumor $(1 / 15,6.7 \%)$ and thyroid $(1 / 15,6.7 \%$; data not shown). The most frequently used markers included the estrogen receptor (ER), progesterone receptor (PR), human epidermal growth factor receptor-2 (HER-2), mammaglobin, gross cystic disease fluid protein-15 (GCDFP-15), cytokeratin, p63, transcription termination factor-1 (TTF-1), S-100 by IHC and Epstein-Barr virus-encoded RNA (EBER) by in situ hybridization, which together with the primary tumor history, were conducive for accurate diagnosis.

Surgical resection of the breast was performed in 17 patients (77.3\%): 7 patients (31.8\%) had modified radical mastectomy and 10 patients $(45.5 \%)$ had breast-conserving surgery (data not shown). Follow-up data were available in 20 cases. The median survival duration from diagnosis of metastases to the breast was 35 months (range, 2-106 months). A total of 10 patients $(45.5 \%)$ succumbed from the disease. Univariate analysis was applied to evaluate different interventions on overall survival (Figs. 6-8). Patients who underwent surgery had a median survival duration of 74 months, while patients who did not undergo surgery survived for a median duration of 12 months $(\mathrm{P}=0.023$; Fig. 7). However, the difference in survival observed among patients who underwent modified radical mastectomy, breast-conserving surgery and non-surgical intervention was not significant $(\mathrm{P}=0.301$; Fig. 8$)$. 


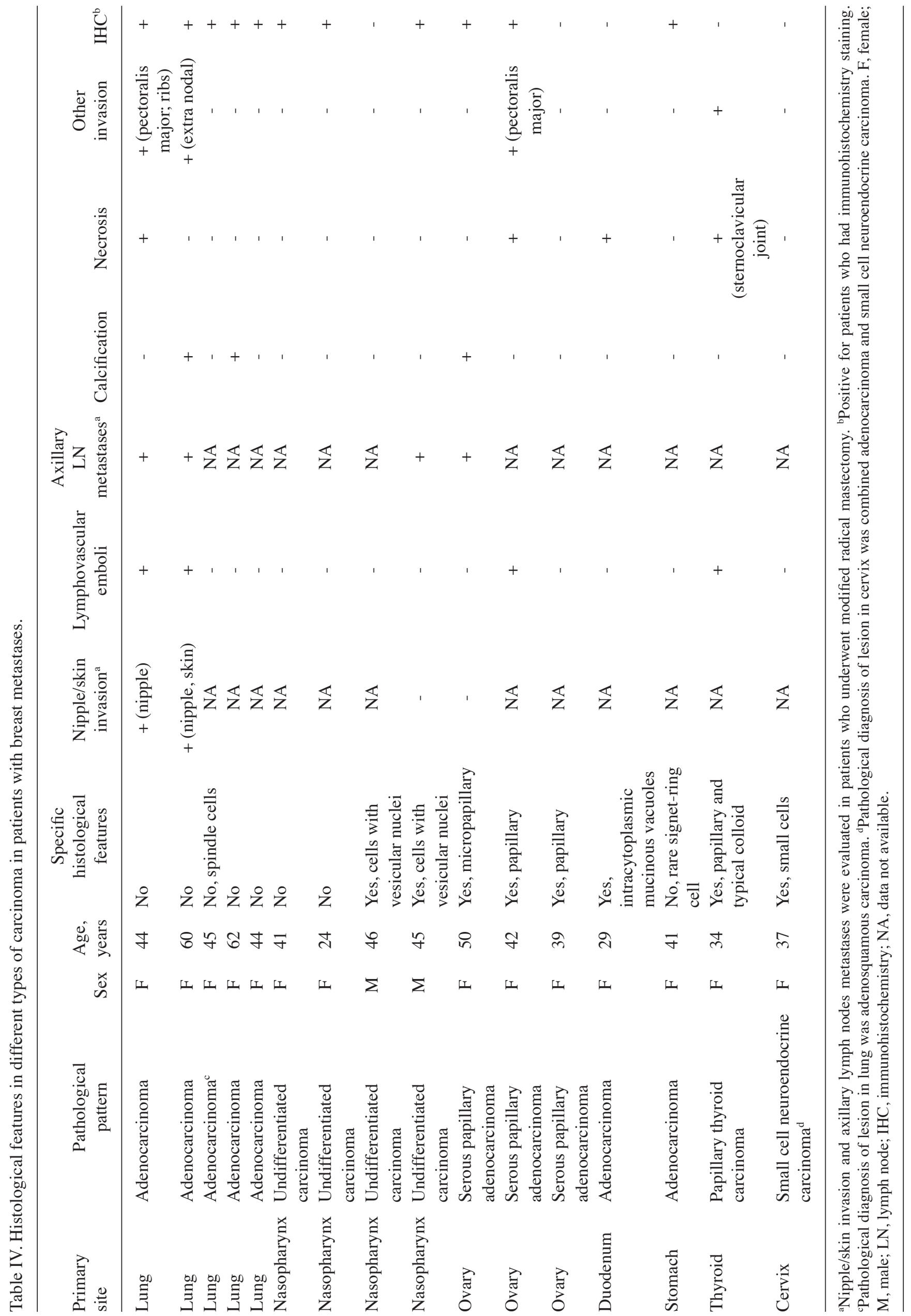




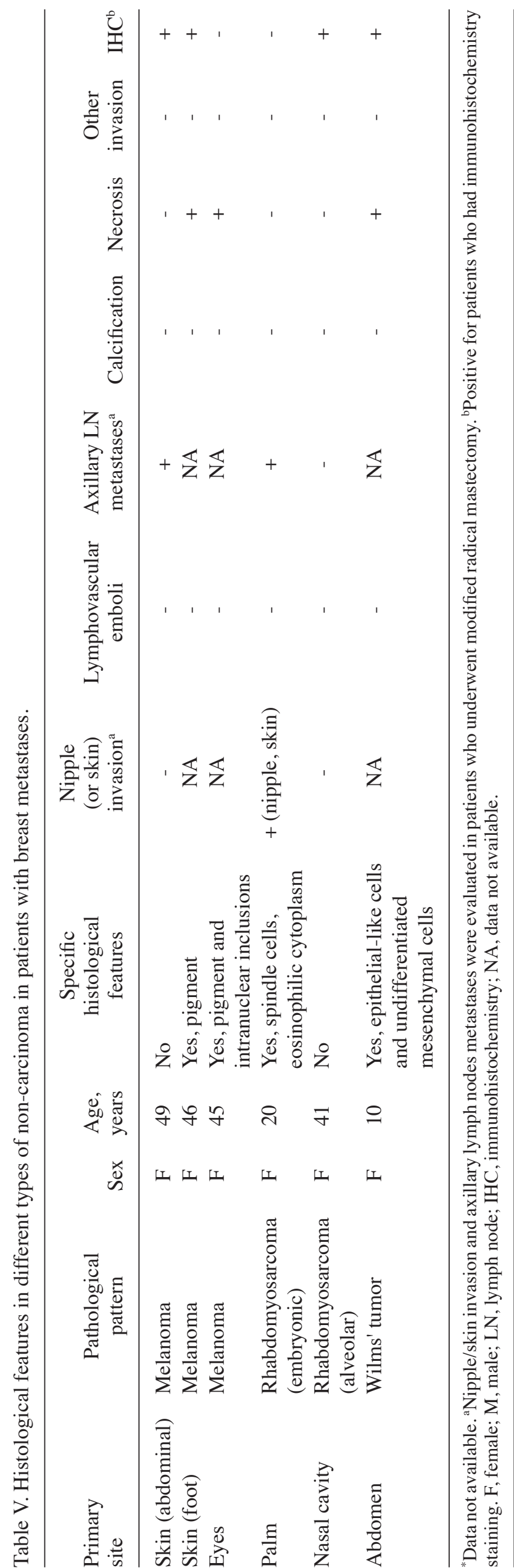

\section{Discussion}

Metastasis to the breast may occur in various types of non-breast solid tumor; however, cases of this are extremely rare $(1-5,10)$. Additionally, the therapeutic strategies used to treat these cases are extremely different from those used to treat primary breast cancer, making the history of the primary tumor particularly important for the achievement of an accurate diagnosis (10). Williams et al (3) and Lee et al (4) demonstrated that a documented history of a primary tumor was observed in $72-88 \%$ of patients with breast metastases, and that these metastases more frequently occurred in females, which is consistent with the results of the present study. The results of the present study also demonstrated that the breast lesion was the initial or only metastasis in half of the patients, which is inconsistent with previous studies (4,10-13), which demonstrated that these metastases are most likely to originate from carcinoma of genital organs $(3 / 4,75.0 \%)$ and non-carcinoma (5/6, 83.3\%). These inconsistencies may be due to the variance in pathological cancer types and the young onset age of 40.5 years in the patients used in the current study, which is an age group that remains at high risk of breast cancer (14). Other previous studies have indicated that there is a higher incidence of metastasis to the breast from non-breast solid tumors that occur in adolescence (15), as well as during lactation and pregnancy (16), owing to the change in hormonal status during these periods. Longer median time (2 years) intervals from the point of diagnosis of the primary tumor to breast metastasis compared with those in the current study have also been reported (17).

A single palpable painless mass located in the upper outer quadrant of the breast was the most common initial clinical feature reported in the current study. Akcay et al (2) demonstrated that the breast metastases were frequently multiple and bilateral, which was observed in $36.4 \%$ (8/22) and 18.2\% (4/22) of patients in the current study, respectively. Laterality was not present at either side of the breast, which was inconsistent with earlier studies $(3,4,17)$. Lee et al (4) suggested that a preponderant lymphatic pathway to the breast from other organs may contribute to laterality; however, further investigations are required. Considering the rarity of breast metastases, a new primary breast cancer may be considered 'preferable' for the breast lesion even in patients with a history of definite primary extramammary malignancy $(3,4,17)$. Radiological imaging also aids the establishment of a more accurate diagnosis (18).

Previous ultrasonic and mammographic images studies demonstrated that it is occasionally difficult to distinguish breast metastases, which appeared as a hypoechoic or high-density well-circumscribed and freely movable mass, from primary breast cancer which displays a hypoechoic mass with speculated margin or a diffuse lesions with or without calcifications $(5,18,19)$. However, despite the absence of diffuse lesions, $42.9 \%$ of the patients in the current study presented with a mass with a speculated margin. Calcifications are rarely observed in breast metastases $(20,21)$, with the exception of metastatic ovarian papillary carcinoma with psammoma bodies $(22,23)$, which is consistent with the results of the current study. Two cases of breast metastasis from lung adenocarcinoma with calcifications was also presented in the current study. Breast lesions were classified as BI-RADS IVA or 
A

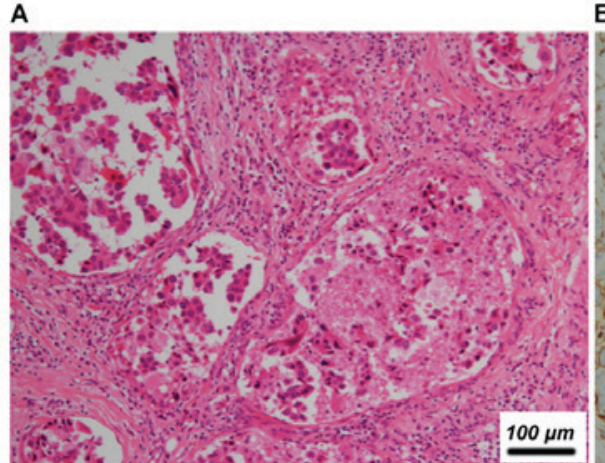

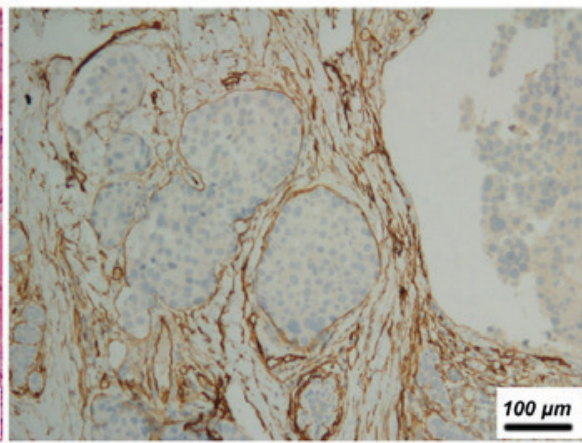

C

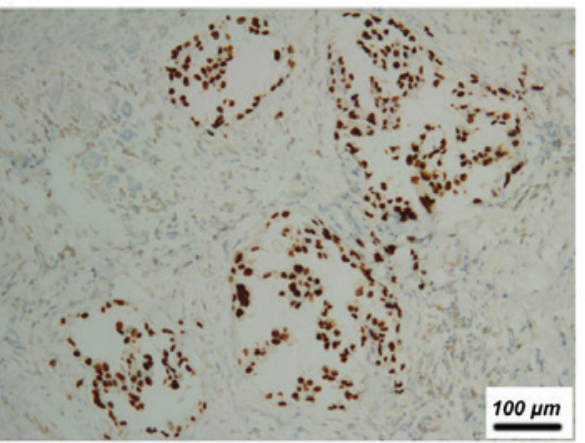

Figure 1. Metastasis from lung adenocarcinoma to the breast. (A) A large quantity of lymphovascular tumor cell embolus with necrosis, which should be distinguished from ductal carcinoma in situ. Immunohistochemical analysis indicated the expression of (B) CD34 in vessels and (C) thyroid transcription factor-1 in tumor cells. Scale bar, $100 \mu \mathrm{m}$; magnification, $\mathrm{x} 10$.

A

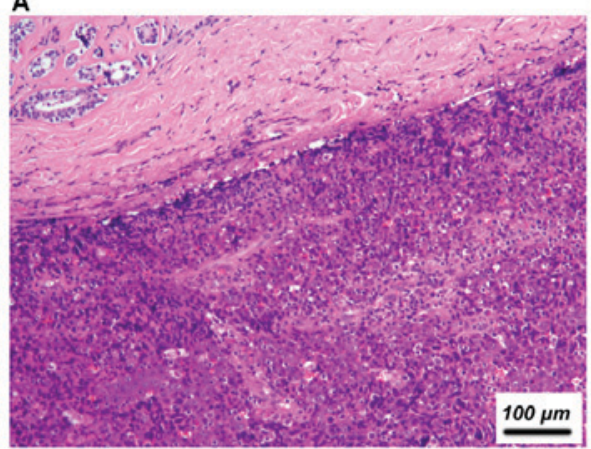

B

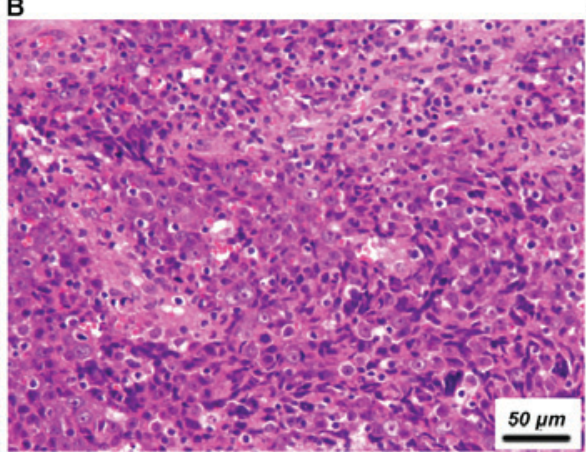

C

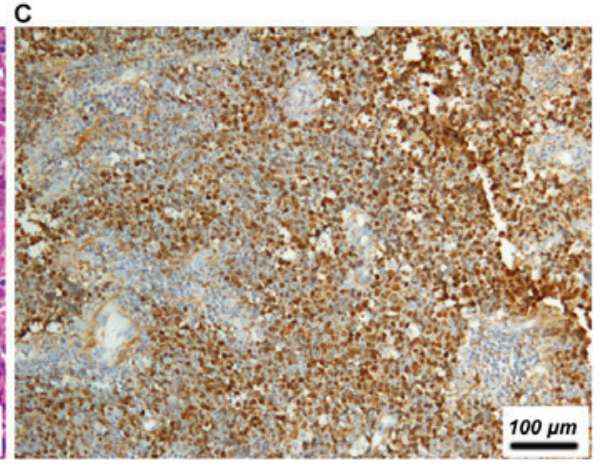

Figure 2. Metastasis from non-keratinizing undifferentiated carcinoma of the nasopharynx to the breast. (A) Well-circumscribed lesion surrounded by a fibrous pseudocapsule in H\&E staining. (B) Typical vesicular nucleus tumor cells with distinct nucleolus without keratinization in H\&E staining at magnification, x20. (C) In situ hybridization (ISH) for detection of Epstein-Barr virus-encoded RNA indicated that the tissues were EBV positive. Scale bar, $100 \mu$ m; magnification, $\mathrm{x} 10$.

A

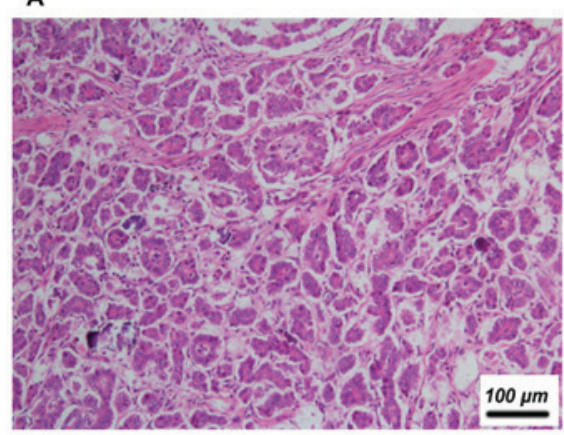

C

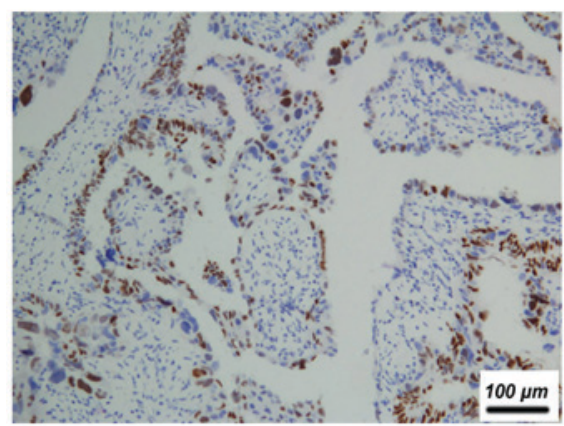

B

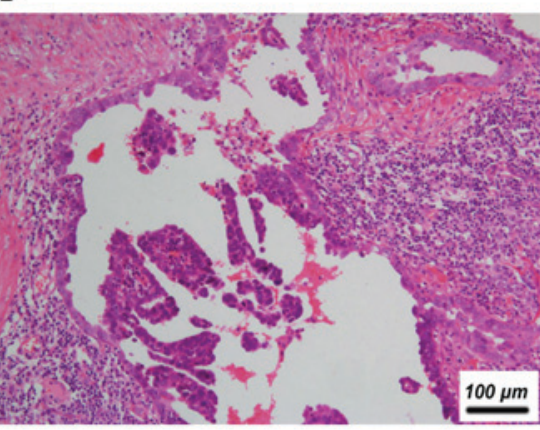

D

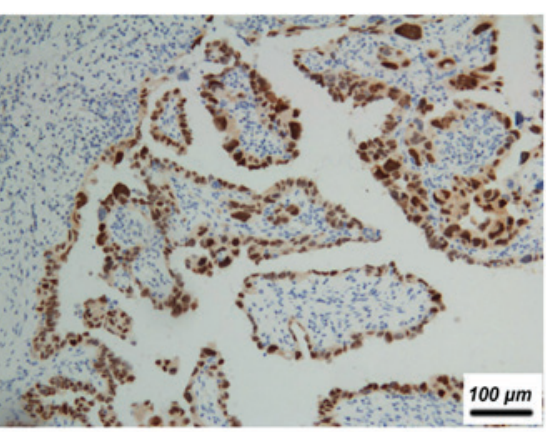

Figure 3. Metastasis from serous papillary carcinoma of the ovary to the breast. (A) Micropapillary with calcification and (B) typical papillary architecture in $\mathrm{H} \& \mathrm{E}$ staining, which should be distinguished from invasive micropapillary carcinoma of the breast. Immunohistochemical staining indicated expression of (C) Wilms' tumor 1 and (D) paired box protein PAX-8 in tumor cell nuclei. Scale bar, $100 \mu \mathrm{m}$; magnification, x10. 
A

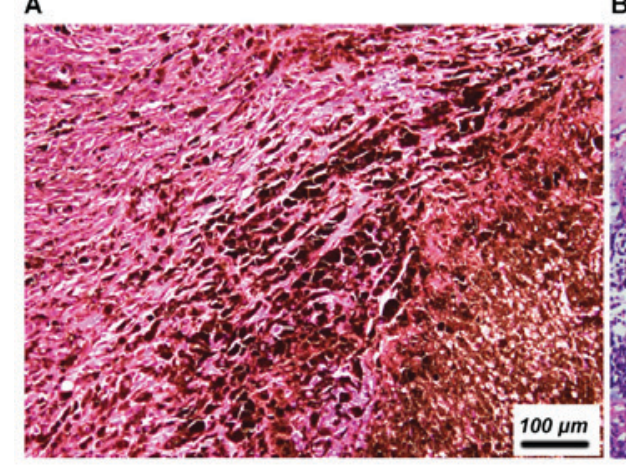

B

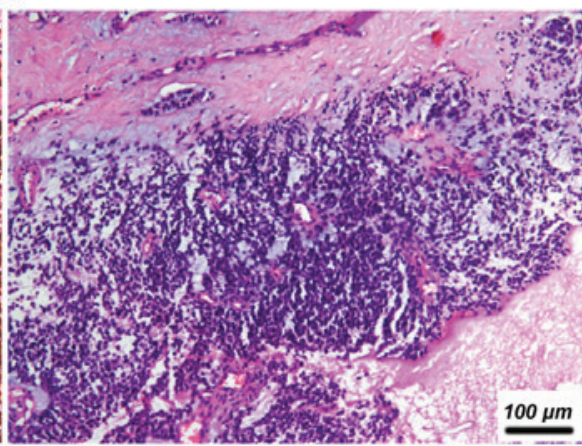

C

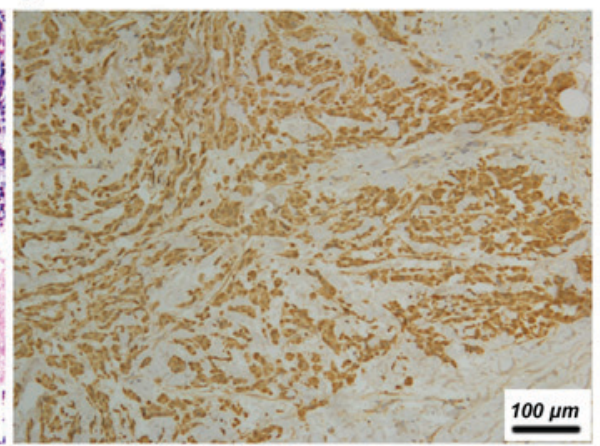

Figure 4. Metastasis from melanoma to the breast. (A) Typical spindle cells and pigment with necrosis in H\&E staining. (B) Atypical small round blue cells with necrosis and myxoid stroma, no pigment is observed in H\&E staining. (C) Immunohistochemical staining indicated S-100 expression. Scale bar, $100 \mu$ m; magnification, $\mathrm{x} 10$.

A

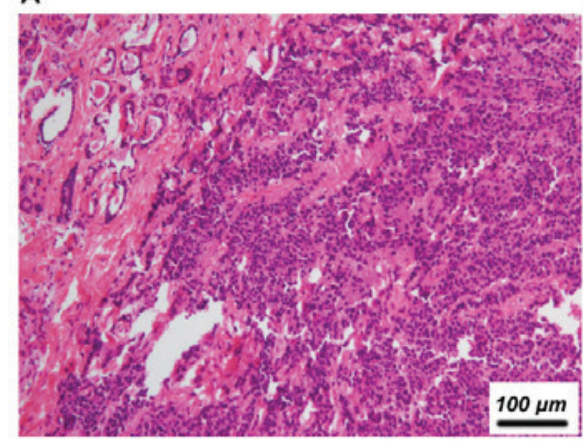

B

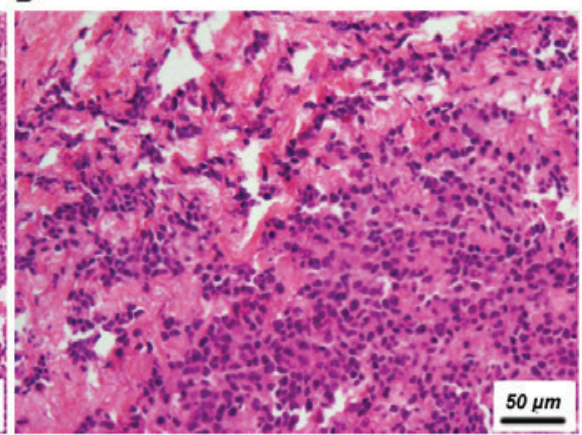

C

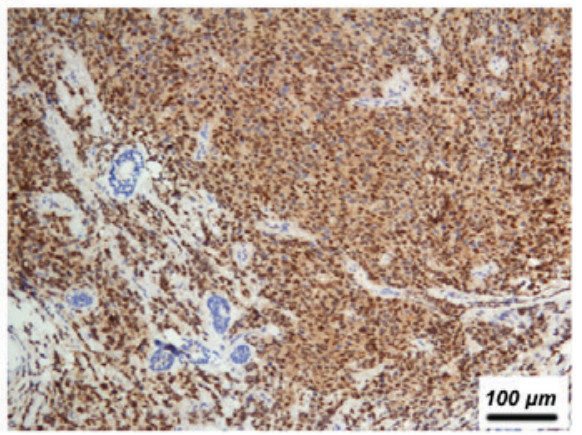

Figure 5. Metastasis from rhabdomyosarcoma to the breast. (A) Typical spindle tumor cells with ill-defined margins and (B) skeletal muscle-like tumor cells characterized by eosinophilic cytoplasm in H\&E staining at magnification, x20. (C) Immunohistochemical staining indicated myogenin expression. Scale bar, $100 \mu \mathrm{m}$; magnification, $\mathrm{x} 10$.

greater in the majority of cases in the present study; the others were categorized as BI-RADS I-III, suggesting that a metastatic mass from a solitary cyst should be distinguished from a metastatic mass from a fibroadenoma, particularly in postmenopausal women or women with a known history of cancer. Posterior echo enhancement and vascularity demonstrated by Color Doppler have previously been used to accurately identify a lesion as either a metastatic melanoma or sarcoma $(5,24)$ and the results of the present study revealed that posterior echoic enhancement and vascularity were observed in all three cases of breast metastases from melanoma. Asian women have smaller breasts with higher gland density and lower lipid content (25) than women from Western countries. The lack of calcification may therefore lead to misdiagnosis of breast lesions by mammography $(5,22)$. Further research comparing the accuracy of ultrasonic screening and mammography for diagnosis of breast metastases in Asian females is required. Computerized tomography and magnetic resonance imaging have also been used for diagnosis of breast metastases $(26,27)$; however, $95 \%$ of the patients in the present study underwent a biopsy followed by radiological imaging, confirming the diagnosis of breast metastasis.

Consistent with previous reports $(3,15,17)$, lung adenocarcinoma, ovarian serous papillary carcinoma and melanoma were the most common primary carcinomas that metastasized to the breast. DeLair et al (17) also reported that lung adenocarcinoma and melanoma were more common in males. Previous studies have reported the relatively high incidence of gastric carcinoma (4) and lymphoma (28). To the best of our knowledge, the present study is the first to report nasopharyngeal carcinoma (NPC) as accounting for a high proportion $(4 / 22)$ of primary tumors, with half of these cases occurring in men. The inconsistency between the results of the present and previous studies may be due to referral (the Sun Yat-sen University Cancer Center is known for its excellence in nasopharyngeal carcinomas care), geographical and/or racial biases, as there is a higher disease prevalence of nasopharyngeal carcinoma in southern Chinese Han males $(29,30)$. Typical morphological features of NPC, including cells with vesicular nuclei and positive in situ hybridization staining for EBERs, as well as immunostaining of cytokeratin 5/6 and p63, aided the achievement of a diagnosis of NPC in the current study. However, given the limited numbers of cases reported thus far, further investigation is required.

Breast metastases and primary breast cancer have common histological findings, including periductal and perilobular distribution, absence of ductal carcinoma in situ, lack of stromal reaction, including desmoplastic response and elastosis and a large number of lymphovascular tumor emboli $(4,15,31)$. The majority of metastatic lesions have histological appearances consistent with their primary sites and thus, pathologists often identify a metastasis to the breast by comparing histological 


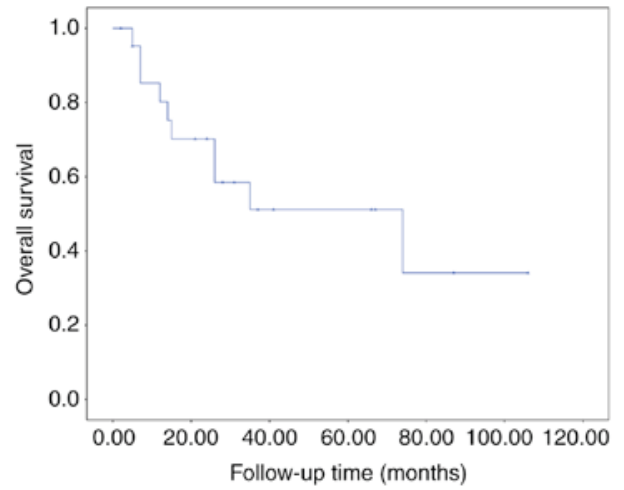

Figure 6. Kaplan Meier analysis of overall survival for patients with metastasis to the breast.

patterns to a previous sample from the patient (4). This evaluation was performed with metastatic melanoma, sarcoma, neuroendocrine carcinoma and Wilms tumor in the present study. Immunohistochemical staining for tumor-specific markers, including ER, PR, HER-2, mammaglobin, GCDFP-15 and GATA binding protein 3 (GATA3) for breast cancer; TTF-1 and Napsin A for lung adenocarcinoma; Wilms tumor protein, paired box protein PAX-8 (PAX-8) and CA-125 for ovarian carcinoma; CK20 and homeobox protein CDX-2 for gastrointestinal tract adenocarcinoma; CgA, NSE and Synapsin for neuroendocrine carcinoma; CK19 and thyroid peroxidase for thyroid carcinoma; S-100 and HMB-45 for melanoma; and myogenin for rhabdomyosarcoma aid diagnosis. However, several overlaps exist, including ER, PR and GCDFP-15 in histological appearance and immunophenotyping, particularly between ovarian carcinoma and primary breast cancer, often leading to difficulties in differential diagnosis (32). Prior studies have reported that GCDFP-15 was rarely observed in ovarian carcinoma $(33,34)$, whereas $~ 30 \%$ of primary breast cancer also did not exhibit GCDFP-15 expression (34). Other studies have reported that up to $95 \%$ of serous papillary carcinomas exhibit nuclear expression of WT-1and membrane expression of CA125, which is only present in $<10 \%$ of different types of breast cancer (35-37). A more recent study also suggested the value of GATA- 3 and PAX- 8 as biomarkers for breast cancer $(38,39)$. Consistent with a previous study $(3)$, the results of the current study indicate that no single marker is absolutely specific and its expression is always variable between primary and metastatic lesions, particularly in tissue biopsies. Thus, a panel of IHC markers with the same pathology as that of the primary tumor, clinical history and imaging findings are required in combination for accurate diagnosis.

The prognosis of metastases to the breast remains poor $(1-4,10,17)$. The median survival duration reported by Williams et al (3) (169 cases), DeLair et al (17) (85 cases) and Lee et al (4) (30 cases) was 10, 15 and 13.9 months, respectively, which is consistent with the results of the current study, in which the shortest survival duration following diagnosis was $>2$ months. Over $70 \%$ of patients had widely metastatic disease in combination with the breast lesion, which is likely to be the main contributor to the poor survival observed in the present study. Individualized systemic therapeutic strategies for primary tumors should be recommended as the primary therapy in a

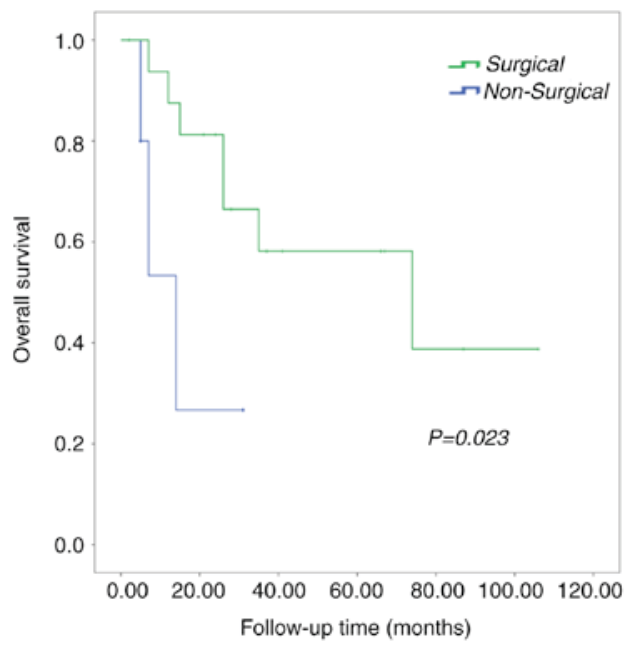

Figure 7. Kaplan Meier analysis of overall survival for patients with metastasis to the breast stratified by surgical and non-surgical intervention.

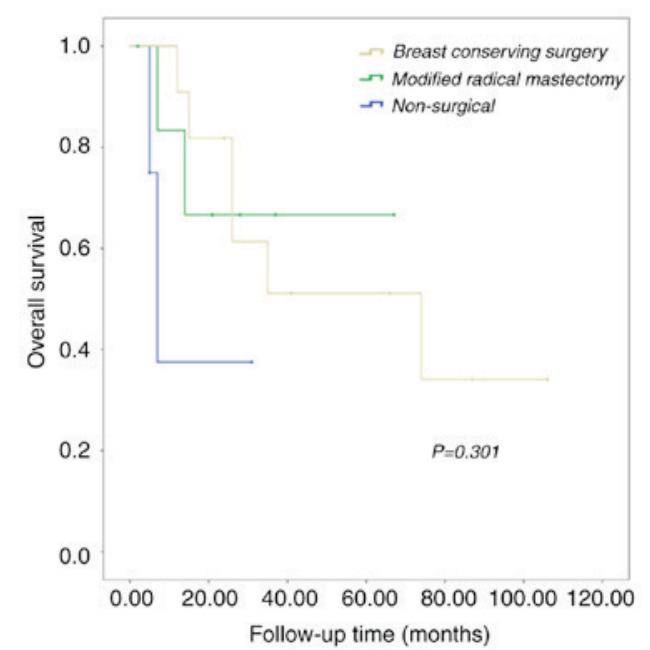

Figure 8. Kaplan Meier analysis of overall survival for patients with metastasis to the breast stratified by breast conserving surgery, modified radical mastectomy and non-surgical intervention.

majority of cases $(1-4,10,17)$; however, the benefit of surgical resection of the breast remains controversial. Consistent with the findings of Williams et al (3), the results of the current study indicated improved overall survival in patients who underwent surgery compared with those who did not. However, there are limitations to the current study: The sample size was extremely small and there was selection bias, as surgery is not well tolerated in patients with advanced disease or poor health condition, as described previously (3). Previously, Rossfeld and Carson (40) proposed the benefit of metastasectomy and suggested the re-evaluation of the approach of 'sparing patients unnecessary surgery'. Understanding the patients' therapeutic goals should be the determinant factor in treating metastatic lesions. Further multi-center clinical investigations are therefore required to address the characteristics of breast metastases that originate from non-breast solid tumor, as well as the effect of surgery on patient prognosis.

In summary, breast metastases are rare and primarily indicate a poor prognosis. Additionally, it may be easily 
misdiagnosed as a primary breast cancer. Clinical manifestations, radiologic findings and histopathological/immunohistochemical features should all be considered in differentiating a secondary mass from a primary breast cancer, even in patients without a history of primary malignant tumors. Early and accurate diagnosis is conducive to individualized treatment and improved prognosis improvement. In addition, surgical resection of breast metastases may result in a survival benefit, which remains yet to be further studied.

\section{References}

1. Alva S and Shetty-Alva N: An update of tumor metastasis to the breast data. Arch Surg 134: 450, 1999.

2. Akçay MN: Metastatic disease in the breast. Breast 11: 526-528, 2002 .

3. Williams SA, Ehlers RA II, Hunt KK, Yi M, Kuerer HM, Singletary SE, Ross MI, Feig BW, Symmans WF and Meric-Bernstam F: Metastases to the breast from nonbreast solid neoplasms: Presentation and determinants of survival. Cancer 110: 731-737, 2007.

4. Lee SK, Kim WW, Kim SH, Hur SM, Kim S, Choi JH, Cho EY, Han SY, Hahn BK, Choe JH, et al: Characteristics of metastasis in the breast from extramammary malignancies. J Surg Oncol 101: 137-140, 2010.

5. Sippo DA, Kulkarni K, Carlo PD, Lee B, Eisner D, Cimino-Mathews A and Harvey SC: Metastatic disease to the breast from extramammary malignancies: A multimodality pictorial review. Curr Probl Diagn Radiol 45: 225-232, 2016.

6. Manini C, Pietribiasi F, Sapino A and Donadio S: Serous cystadenocarcinoma of the ovary with simultaneous breast metastases. Description of a case. Pathologica 90: 152-155, 1998 (In Italian).

7. Hanna NN, O'Donnell K and Wolfe GR: Alveolar soft part sarcoma metastatic to the breast. J Surg Oncol 61: 159-162, 1996

8. Oksüzoğlu B, Abali H, Güler N, Baltali E and Ozişik Y: Metastasis to the breast from nonmammarian solid neoplasms: A report of five cases. Med Oncol 20: 295-300, 2003.

9. Liberman L and Menell JH: Breast imaging reporting and data system (BI-RADS). Radiol Clin North Am 40: 409-430, v, 2002.

10. Hajdu SI and Urban JA: Cancers metastatic to the breast. Cancer 29: 1691-1696, 1972.

11. Hamby LS, McGrath PC, Cibull ML and Schwartz RW: Gastric carcinoma metastatic to the breast. J Surg Oncol 48: 117-121, 1991.

12. Toombs BD and Kalisher L: Metastatic disease to the breast: Clinical, pathologic, and radiographic features. AJR Am J Roentgenol 129: 673-676, 1977.

13. Mihai R, Christie-Brown J and Bristol J: Breast metastases from colorectal carcinoma. Breast 13: 155-158, 2004.

14. Brouckaert O, Rudolph A, Laenen A, Keeman R, Bolla MK, Wang Q, Soubry A, Wildiers H, Andrulis IL, Arndt V, et al: Reproductive profiles and risk of breast cancer subtypes: A multi-center case-only study. Breast Cancer Res 19: 119, 2017.

15. Vergier B, Trojani M, de Mascarel I, Coindre JM and Le Treut A: Metastases to the breast: Differential diagnosis from primary breast carcinoma. J Surg Oncol 48: 112-116, 1991.

16. Nayar M, Chandra M, Aggarwal R and Chander S: Carcinoma cervix presenting as primary breast malignancy. Indian J Pathol Microbiol 30: 283-286, 1987.

17. DeLair DF, Corben AD, Catalano JP, Vallejo CE, Brogi E and Tan LK: Non-mammary metastases to the breast and axilla: A study of 85 cases. Mod Pathol 26: 343-349, 2013.

18. Yeh CN, Lin $\mathrm{CH}$ and Chen MF: Clinical and ultrasonographic characteristics of breast metastases from extramammary malignancies. Am Surg 70: 287-290, 2004.

19. Mun SH, Ko EY, Han BK, Shin JH, Kim SJ and Cho EY: Breast metastases from extramammary malignancies: Typical and atypical ultrasound features. Korean J Radiol 15: 20-28, 2014.

20. Bohman LG, Bassett LW, Gold RH and Voet R: Breast metastases from extramammary malignancies. Radiology 144: 309-312, 1982.

21. McCrea ES, Johnston C and Haney PJ: Metastases to the breast. AJR Am J Roentgenol 141: 685-690, 1983.
22. Lee SH, Park JM, Kook SH, Han BK and Moon WK: Metastatic tumors to the breast: Mammographic and ultrasonographic findings. J Ultrasound Med 19: 257-262, 2000.

23. Vizcaíno I, Torregrosa A, Higueras V, Morote V, Cremades A Torres V, Olmos S and Molins C: Metastasis to the breast from extramammary malignancies: A report of four cases and a review of literature. Eur Radiol 11: 1659-1665, 2001.

24. Surov A, Fiedler E, Holzhausen HJ, Ruschke K, Schmoll HJ and Spielmann RP: Metastases to the breast from non-mammary malignancies: Primary tumors, prevalence, clinical signs, and radiological features. Acad Radiol 18: 565-574, 2011.

25. Torjesen I: Adding ultrasound to mammography could increase breast cancer detection in Asian women. BMJ 351: h5926, 2015.

26. Phadke S, Thomas A, Yang L, Moore C, Xia C and Schroeder MC: Frequency and clinical significance of extramammary findings on breast magnetic resonance imaging. Clin Breast Cancer 16: 424-429, 2016 .

27. Benveniste AP, Marom EM, Benveniste MF, Mawlawi OR, Miranda RN and Yang W: Metastases to the breast from extramammary malignancies-PET/CT findings. Eur J Radiol 83: 1106-1112, 2014.

28. Buisman FE, van Gelder L, Menke-Pluijmers MB, Bisschops BH, Plaisier PW and Westenend PJ: Non-primary breast malignancies: A single institution's experience of a diagnostic challenge with important therapeutic consequences-a retrospective study. World J Surg Oncol 14: 166, 2016.

29. Leach BI, Sun B, Petrovic L and Liu SV: Breast metastasis from nasopharyngeal carcinoma: A case report and review of the literature. Oncol Lett 5: 1859-1861, 2013.

30. Li S and Yang J: Nasopharyngeal carcinoma metastasis to the mammary gland: A case report. Oncol Lett 9: 275-277, 2015.

31. Georgiannos SN, Chin J, Goode AW and Sheaff M: Secondary neoplasms of the breast: A survey of the 20th century. Cancer 92: 2259-2266, 2001

32. Tornos C, Soslow R, Chen S, Akram M, Hummer AJ, Abu-Rustum N, Norton L and Tan LK: Expression of WT1, CA 125 , and GCDFP-15 as useful markers in the differential diagnosis of primary ovarian carcinomas versus metastatic breast cancer to the ovary. Am J Surg Pathol 29: 1482-1489, 2005.

33. Dennis JL, Hvidsten TR, Wit EC, Komorowski J, Bell AK, Downie I, Mooney J, Verbeke C, Bellamy C, Keith WN and Oien KA: Markers of adenocarcinoma characteristic of the site of origin: Development of a diagnostic algorithm. Clin Cancer Res 11: 3766-3772, 2005.

34. Moritani S, Ichihara S, Hasegawa M, Endo T, Oiwa M, Yoshikawa K, Sato Y, Aoyama H, Hayashi T and Kushima R: Serous papillary adenocarcinoma of the female genital organs and invasive micropapillary carcinoma of the breast. Are WT1, CA125, and GCDFP-15 useful in differential diagnosis? Hum Pathol 39: 666-671, 2008.

35. Lagendijk JH, Mullink H, van Diest PJ, Meijer GA and Meijer CJ: Immunohistochemical differentiation between primary adenocarcinomas of the ovary and ovarian metastases of colonic and breast origin. Comparison between a statistical and an intuitive approach. J Clin Pathol 52: 283-290, 1999.

36. Recine MA, Deavers MT, Middleton LP, Silva EG and Malpica A: Serous carcinoma of the ovary and peritoneum with metastases to the breast and axillary lymph nodes: A potential pitfall. Am J Surg Pathol 28: 1646-1651, 2004.

37. Lee BH, Hecht JL, Pinkus JL and Pinkus GS: WT1, estrogen receptor, and progesterone receptor as markers for breast or ovarian primary sites in metastatic adenocarcinoma to body fluids. Am J Clin Pathol 117: 745-750, 2002.

38. Espinosa I, Gallardo A, D'Angelo E, Mozos A, Lerma E and Prat J: Simultaneous carcinomas of the breast and ovary: Utility of Pax-8, WT-1, and GATA3 for distinguishing independent primary tumors from metastases. Int J Gynecol Pathol 34: 257-265, 2015.

39. Tempfer CB, El Fizazi N, Ergonenc H and Solass W: Metastasis of ovarian cancer to the breast: A report of two cases and a review of the literature. Oncol Lett 11: 4008-4012, 2016.

40. Rossfeld KK and Carson WE III: Surgical management of ovarian carcinoma metastatic to the breast and axilla: A role for metastasectomy? J Surg Oncol 112: 581-584, 2015.

This work is licensed under a Creative Commons Attribution-NonCommercial-NoDerivatives 4.0 International (CC BY-NC-ND 4.0) License. 